\title{
Loss of SerpinA5 protein expression is associated with advanced-stage serous ovarian tumors
}

Ingrid TGW Bijsmans ${ }^{1}$, Kim M Smits ${ }^{1}$, Pauline de Graeff ${ }^{2}$, G Bea A Wisman ${ }^{2}$, Ate GJ van der Zee ${ }^{2}$, Brigitte F Slangen ${ }^{3}$, Adriaan P de Bruïne ${ }^{1}$, Manon van Engeland ${ }^{1}$, Nathalie L Sieben ${ }^{1}$ and Koen K Van de Vijver $^{1}$

${ }^{1}$ Department of Pathology, GROW, School for Oncology and Developmental Biology, Maastricht University Medical Center, Maastricht, The Netherlands; ${ }^{2}$ Department of Gynecological Oncology, University Medical Center Groningen and University of Groningen, Groningen, The Netherlands and ${ }^{3}$ Department of Obstetrics and Gynecology, GROW, School for Oncology and Developmental Biology, Maastricht University Medical Center, Maastricht, The Netherlands

Epithelial ovarian cancer, the most lethal neoplasm of the female genital tract, is usually diagnosed at an advanced stage as obvious symptoms are absent at early stages. This disease is believed to originate from malignant transformation of the ovarian surface epithelium or fallopian tube. Histologically, several subtypes are being recognized, with serous histology accounting for the majority of cases. Serous tumors include serous borderline tumors and serous carcinomas. A better understanding of the tumor biology and molecular mechanisms involved in these tumors is needed, as both patient management and prognosis differ substantially. Previous microarray analysis identified SerpinA5, a uPA inhibitor, as key regulator for indolent borderline behavior. As carcinomas are characterized by loss of SerpinA5 mRNA expression, we hypothesized that SerpinA5 protein expression is reduced or lost in carcinomas when compared with borderline tumors. We performed SerpinA5 immunohistochemical staining on 32 serous borderline tumors, 187 primary serous carcinomas and 62 serous omental metastases. Reduced or absent SerpinA5 protein staining was observed in carcinomas when compared with borderline tumors $(P<0.001)$. SerpinA5 protein expression was significantly lowered in the omental metastases $(P<0.001)$ when compared with the matching primary carcinoma. Interestingly, SerpinA5 protein expression was reduced in advanced-stage borderline tumors, often characterized by micropapillary growth and/or microinvasion, when compared with early-stage borderline tumors $(P=0.015)$. In conclusion, SerpinA5 expression is significantly reduced in advanced-stage serous borderline tumors and serous carcinomas when compared with the early-stage counterparts, and reduction of expression is linked to more aggressive features of borderline tumors.

Modern Pathology (2011) 24, 463-470; doi:10.1038/modpathol.2010.214; published online 19 November 2010

Keywords: immunohistochemistry; PCl; serous borderline tumor; serous ovarian carcinoma; SerpinA5; tissue microarray

Surface epithelial stromal tumors of the ovary are a leading cause of death among women suffering from cancer of the female genital tract. This disease is usually diagnosed at an advanced stage, as obvious

Correspondence: Dr KK Van de Vijver, MD, PhD, Department of Pathology, Maastricht University Medical Center, GROW, School for Oncology and Developmental Biology, PO Box 5800, 6202 AZ Maastricht, The Netherlands.

E-mail: koen.vande.vijver@mumc.nl

Received 30 August 2010; revised 14 October 2010; accepted 14

October 2010; published online 19 November 2010 symptoms are absent at early stages. Worldwide, ovarian cancer was estimated at 225000 cases and 140000 deaths in 2008. ${ }^{1}$ Epithelial ovarian cancer is believed to be caused by malignant transformation of the ovarian surface epithelium. ${ }^{2}$ However, current evidence suggests that high-grade serous carcinomas might originate from the fallopian tube. ${ }^{3-5}$ Serous borderline tumors belong to an intermediate group of ovarian tumors, characterized by arborizing papillae lined by stratified epithelial cells, varying atypia and lack of destructive stromal invasion. In all, $30 \%$ of serous borderline tumor patients 
present with tumor deposits, the so-called implants, on peritoneal surfaces, omentum and in lymph nodes. However, the 5-year survival remains excellent ( $>90 \%)^{2,6,7}$ In contrast, serous carcinomas show obvious stromal invasion associated with an average 5 -year survival of $25 \% .^{6,8}$

Recently, the molecular mechanisms involved in borderline tumor and carcinoma development are being unraveled. BRAF and KRAS mutations are frequently detected in serous borderline tumors. ${ }^{9,10}$ This was the first clue indicating that the RAS signaling pathway is important in tumorigenesis of serous borderline tumors. TP53 mutations are present in up to $80 \%$ of serous carcinomas, whereas they are almost absent in serous borderline tumors. ${ }^{6,11}$ Microarray expression profiling studies have confirmed that borderline tumors and carcinomas represent different disease entities. ${ }^{12-16} \mathrm{We}$ identified a putative tumor-suppressor gene Serpi$n A 5$, also referred to as plasminogen activator inhibitor-3 (PAI-3) or protein $C$ inhibitor (PCI), as being differentially expressed between serous borderline tumors and carcinomas. ${ }^{16}$ SerpinA5 is a member of the serine protease inhibitor superfamily $^{17}$ that inactivates a variety of plasma proteases, including urokinase-type plasminogen activator (uPA), anticoagulant serine proteases, blood coagulation factors and fibrinolytic enzymes. ${ }^{18}$ The presence of SerpinA5 in serous borderline tumors indicates that despite active RAS signaling, activation of downstream genes involved in extracellular matrix degradation is absent, whereas loss of SerpinA5 mRNA expression in serous carcinomas was associated with downstream activation of matrix metalloproteinase 9 (MMP9), ${ }^{16}$ explaining the invasive potential of serous carcinomas because of degradation of the extracellular matrix.

Additional lines of evidence indicate that SerpinA5 is associated with invasiveness. SerpinA5 expression is decreased in renal, prostate and ovarian cancers. ${ }^{16,19,20}$ Overexpression of SerpinA5 results in decreased invasion, metastatic potential and angiogenesis in breast cancer, ${ }^{21}$ and elevated levels of SerpinA5 protein was reported to be associated with a better survival in primary breast carcinomas. ${ }^{22}$ The aim of this study was to investigate the relation between SerpinA5 protein expression and histopathological characteristics in serous borderline tumors and carcinomas by immunohistochemistry.

\section{Materials and methods}

\section{Patients and Pathologic Review}

Formalin-fixed paraffin-embedded tissue of 76 serous tumors was obtained from the archives of the Departments of Pathology, Maastricht University Medical Center (Maastricht, The Netherlands) and Stichting PAMM (Eindhoven, The Netherlands), collectively referred to as Maastricht series. Tissue microarrays of 177 primary serous carcinomas and 88 omental metastases were obtained from the Department of Gynecologic Oncology, University Medical Center Groningen (Groningen, The Netherlands). ${ }^{23}$ Review of hematoxylin and eosin (H\&E) slides was performed for consensus diagnosis by two experienced gynecologic pathologists (NLS, KKVdV). Serous tumors were considered invasive (= serous carcinoma) if destructive stromal invasion was present. Serous carcinomas were divided into low grade (grade 1) and high grade (grades 2 and 3 ). Micropapillary growth pattern was characterized by a filigree pattern of highly complex micropapillae growing non-hierarchically. In serous borderline tumors, microinvasion was defined as the presence of single cells or nests of cells in the stroma. ${ }^{6}$ The Maastricht series consisted of 32 serous borderline tumors ( 3 with microinvasion, 7 with micropapillary growth and 1 with both microinvasion and micropapillary growth) and 44 serous carcinomas (5 grade 1, 14 grade 2 and 25 grade 3 serous carcinomas). None of the patients received chemotherapy before surgery (see Table 1 for detailed clinicopathological characteristics and Figure 1 for the characteristics of the populations). Collection, storage and use of tissue and patient data were performed in agreement with the 'Code for Proper Secondary Use of Human Tissue in the Netherlands' (http://www.fmwv.nl). This study was approved by the medical ethical committee of the Maastricht University Medical Center, The Netherlands.

Statistically significant differences between the serous carcinomas derived from the Maastricht and Groningen series were not identified for the baseline characteristics tumor grade, FIGO (International Federation of Gynecology and Obstetrics) stage, unilateral versus bilateral tumors and age at diagnosis (data not shown). Therefore, these were considered as one population.

At the end of this study, 36 patients ( 24 borderline tumors and 12 carcinomas) of the Maastricht series were alive. Patients who died within 5 years after diagnosis, and of whom autopsy was not performed, were considered 'dead of disease' if no other cause of death was specified. In all, 27 patients (1 borderline tumor and 26 carcinomas) died of disease, 3 ( 2 borderline tumors and 1 carcinoma) died of unrelated causes and 10 (5 borderline tumors and 5 carcinomas) were lost to follow-up. From the 177 cases from Groningen, 71 patients were alive (28 with evidence of disease and 43 without), 105 died (101 cancer related and 4 unrelated) and the outcome of 1 patient was missing.

\section{Serpin A5 Immunohistochemical Staining and Slide Evaluation}

Rabbit anti-human SerpinA5 polyclonal antibody was obtained from Proteintech Group (Bioconnect, 
Table 1 Patient characteristics

\begin{tabular}{|c|c|c|c|c|}
\hline Characteristics & & $\begin{array}{l}\text { Maastricht } \\
\text { series } \\
(\mathrm{n}=76) \\
\mathrm{n}(\%)\end{array}$ & $\begin{array}{c}\text { Groningen series, } \\
\text { primary serous } \\
\text { carcinoma }(\mathrm{n}=143)^{\mathrm{a}} \\
\mathrm{n}(\%)\end{array}$ & $\begin{array}{l}\text { Groningen series, matched } \\
\text { primary serous carcinoma } \\
\text { and omental metastasis }(\mathrm{n}=62)^{\mathrm{a}}\end{array}$ \\
\hline & Serous borderline tumor & $32(42)$ & & \\
\hline & Serous carcinoma grade 1 & $5(7)$ & $20(14)$ & $3(5)$ \\
\hline & Serous carcinoma grade 2 & $14(18)$ & $43(30)$ & $18(29)$ \\
\hline & Serous carcinoma grade 3 & $25(33)$ & $80(56)$ & $41(66)$ \\
\hline \multicolumn{5}{|l|}{ Age at diagnosis } \\
\hline \multirow{4}{*}{ Years (range) } & Serous borderline tumor & $49.9(19-89)$ & & \\
\hline & Serous carcinoma grade 1 & $53.0(26-75)$ & $52.7(21-78)$ & $66.7(60-78)$ \\
\hline & Serous carcinoma grade 2 & $56.8(37-80)$ & $59.9(22-84)$ & $60.1(22-84)$ \\
\hline & Serous carcinoma grade 3 & $65.5(43-79)$ & $57.6(36-84)$ & $55.7(36-81)$ \\
\hline \multicolumn{5}{|l|}{ FIGO stage $^{\mathrm{b}}$} \\
\hline \multirow{3}{*}{ Serous borderline tumor } & Stage I & $22(71)$ & & \\
\hline & Stage II & $1(3)$ & & \\
\hline & Stage III & $8(26)$ & & \\
\hline \multirow[t]{4}{*}{ Serous carcinoma } & Stage I & $6(14)$ & $10(7)$ & 0 \\
\hline & Stage II & $2(5)$ & $12(9)$ & 0 \\
\hline & Stage III & $33(75)$ & $98(69)$ & $51(82)$ \\
\hline & Stage IV & $3(7)$ & $22(16)$ & $11(18)$ \\
\hline
\end{tabular}

${ }^{\mathrm{a}}$ In this table, only those patients in whom SerpinA5 immunohistochemical staining was successful are included.

${ }^{\mathrm{b}}$ FIGO stage was unavailable for one serous borderline tumor and one carcinoma.

Huissen, The Netherlands). One representative formalin-fixed paraffin-embedded tumor block was selected for immunohistochemical staining. Tissue sections were deparaffinized and incubated for $15 \mathrm{~min}$ in $0.6 \%$ hydrogen peroxide-methanol to block endogenous peroxidase. Antigen retrieval was performed in Tris-EDTA buffer, pH 9.0 (Klinipath, Duiven, The Netherlands) in a microwave oven, followed by cooling in buffer for $30 \mathrm{~min}$. Sections were blocked with 5\% BSA for $30 \mathrm{~min}$. Slides were incubated with primary antibody (1:250) for $60 \mathrm{~min}$, followed by $30 \mathrm{~min}$ of incubation with poly-HRP-anti-mouse/rabbit/rat IgG (Immunologic, Duiven, The Netherlands). Liquid DAB (Dako, Carpinteria, CA, USA) was applied for $15 \mathrm{~min}$. Slides were counterstained with hematoxylin and mounted. As a negative control, primary antibody was omitted. Evaluation of full and tissue microarray sections was performed by two observers (ITGWB and KKVdV) without previous knowledge of clinicopathological characteristics. Discrepant scores were re-evaluated to reach consensus. Cytoplasmic staining of epithelial cells was considered positive. For scoring tumor sections spotted on TMAs, at least two out of four cores for each tumor had to be available; otherwise, the sample was scored as 'not available'. If tumor cells were not present in more than two cores, a sample was scored as 'not determined'. Slides lacking protein expression were scored as negative. The percentage of cells showing SerpinA5 protein expression was graded $<10 \%$ (range: zero to focal staining),
$11-40 \%$ (up to $40 \%$ positive stained tumor cells) or $>40 \%$. Staining of blood vessels was used as an internal positive control. If heterogeneity in staining pattern within one tumor section was observed, the average score of this section was determined. Mean staining intensity of samples spotted on TMAs was taken from 2 to 4 core biopsies.

\section{Data Analysis}

Differences between baseline characteristics, protein expression in serous borderline tumors and carcinomas, primary tumors and omental metastases, and carcinomas from the Maastricht and Groningen series, were determined by Pearson's $\chi^{2}$ test and $t$-test where appropriate (SPSS 15.0 software). Cancer-specific survival was defined as the time from diagnosis until cancer-related death or end of follow-up. Kaplan-Meier curves and log-rank tests were used to estimate the influence of SerpinA5 expression on cause-specific survival. Hazard ratios and corresponding 95\% confidence intervals were assessed by Cox proportional hazard analyses adjusted for age at diagnosis, tumor grade, FIGO stage and tumor limited to one or both ovaries, which are possible confounders known as prognostic factors for ovarian cancer and possibly influencing the crude hazard ratio. Analysis was also performed after stratification for age. The proportional hazard assumption was tested using the Schoenfeld residuals and the log (-log) hazard plots. 


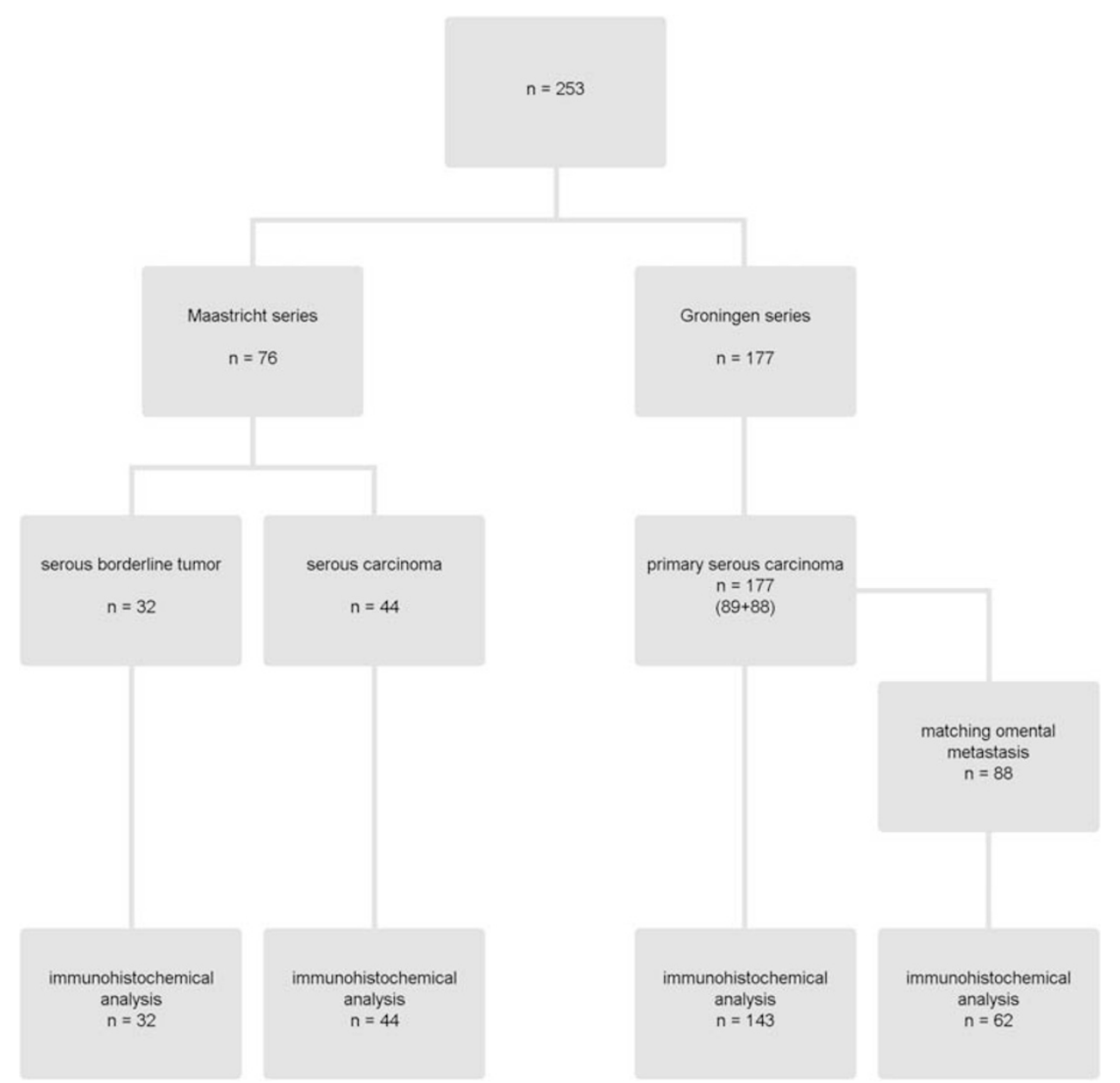

Figure 1 Flowchart of patient populations and SerpinA5 immunohistochemical analysis, representing the number of samples in which SerpinA5 immunohistochemical staining was successful. Patients were excluded if $<2$ tissue cores contained tumor cells or $>2$ cores were nonevaluable because of core loss during the staining process.

Survival analyses were done using STATA 15.0. All $P$-values were two sided and $P \leq 0.05$ were considered statistically significant.

\section{Results}

SerpinA5 protein expression was decreased in serous carcinomas when compared with borderline tumors $(P<0.001$; Table 2). This is in concordance with previous results showing decreased mRNA expression. ${ }^{16}$ Serous borderline tumors expressed SerpinA5 protein in all cases, with the majority of cases $(22 / 32 ; 69 \%)$ showing $>40 \%$ staining. This is in contrast to serous carcinomas that showed $<10 \%$ expression (defined as no or focal protein expression) for most cases (28/44; 64\%). Comparing SerpinA5 protein expression between serous borderline tumors and low-grade serous carcinomas, as well as between serous borderline tumors and high-grade serous carcinomas, showed a statistically significant reduction $(P<0.001)$ in both groups of carcinomas. A representative SerpinA5 staining is shown in Figure 2.
The Groningen series was used as a second, independent patient series for validation of data obtained from the Maastricht series. Successful SerpinA5 staining results were obtained in 143/177 $(81 \%)$ of serous carcinomas. A total of 34 carcinomas were excluded from analysis because less than two tissue cores contained tumor cells $(n=10)$, or more than two cores were non-evaluable because of core loss during the staining process $(n=24)$. SerpinA5 protein expression in serous carcinomas (Table 2) was not statistically different $(P=0.340)$ between the Maastricht and Groningen series: Table 2 shows that 28/44 (64\%) carcinomas of the Maastricht series show $<10 \%$ expression, which is in concordance with the 81/143 (57\%) carcinomas of the Groningen series, thereby confirming loss or decrease of SerpinA5 protein expression in serous carcinomas.

No difference was seen in SerpinA5 protein expression when stratifying the serous carcinomas for FIGO stage, but when comparing the 25 lowgrade carcinomas with the 162 high-grade carcinomas, we detected a statistically significant higher SerpinA5 protein expression in the low-grade 
Table 2 SerpinA5 protein expression in serous borderline tumors and carcinomas

\begin{tabular}{|c|c|c|c|c|c|c|}
\hline \multirow[b]{2}{*}{ Hospital } & \multirow[b]{2}{*}{ Tumor (n) } & & & \multicolumn{3}{|c|}{ Staining score, n (\%) } \\
\hline & & & & $<10 \%$ & $11-40 \%$ & $>40 \%$ \\
\hline \multirow[t]{10}{*}{ Maastricht series } & Serous borderline tumor (32) & & & $3(9)$ & $7(22)$ & $22(69)$ \\
\hline & & Type & Typical (21) & $1(5)$ & $4(19)$ & $16(76)$ \\
\hline & & & Micropapillary and/or microinvasive (11) & $2(18)$ & $3(27)$ & $6(55)$ \\
\hline & & FIGO $^{\mathrm{a}}$ & Early (23) & $1(4)$ & $3(13)$ & $19(83)$ \\
\hline & & & Advanced (8) & $2(25)$ & $4(50)$ & $2(25)$ \\
\hline & Serous carcinoma (44) & & & $28(64)$ & $13(30)$ & $3(7)$ \\
\hline & & Grade & Low-grade serous carcinoma (5) & $2(40)$ & $2(40)$ & $1(20)$ \\
\hline & & & High-grade serous carcinoma (39) & $26(67)$ & $11(28)$ & $2(5)$ \\
\hline & & FIGO & Early (8) & $6(75)$ & $1(13)$ & $1(13)$ \\
\hline & & & Advanced (36) & $22(61)$ & $12(33)$ & $2(6)$ \\
\hline \multirow[t]{7}{*}{ Groningen series } & Serous carcinoma (143) & & & $81(57)$ & $40(28)$ & $22(15)$ \\
\hline & & Grade & Low-grade serous carcinoma (20) & $7(35)$ & $9(45)$ & $4(20)$ \\
\hline & & & High-grade serous carcinoma (123) & $74(60)$ & $31(25)$ & $18(15)$ \\
\hline & & FIGO $^{\text {a }}$ & Early (22) & $10(46)$ & $7(32)$ & $5(23)$ \\
\hline & & & Advanced (120) & $71(59)$ & $32(27)$ & $17(14)$ \\
\hline & Paired (62) & & Serous carcinoma & $35(57)$ & $21(34)$ & $6(10)$ \\
\hline & & & Omental tissue & $45(73)$ & $13(21)$ & $4(7)$ \\
\hline
\end{tabular}

${ }^{\mathrm{a}}$ FIGO stage was unavailable for one serous borderline tumor with micropapillary growth and one serous carcinoma.
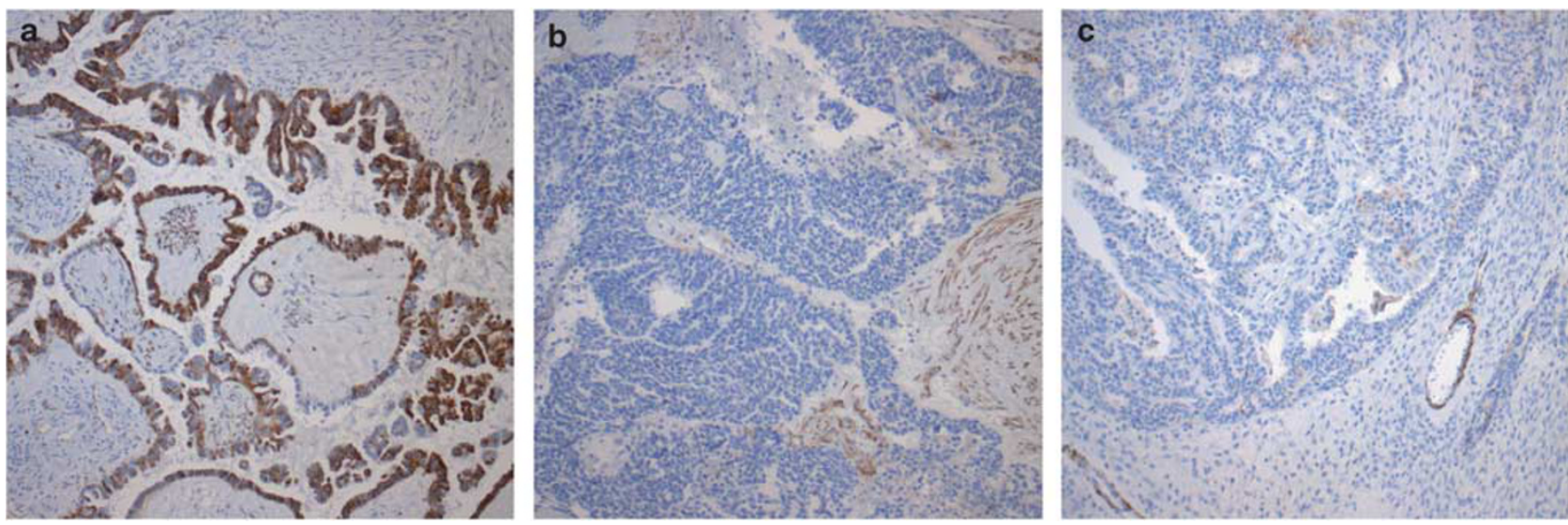

Figure 2 SerpinA5 protein expression in serous borderline tumor and carcinomas. Representative immunohistochemical staining of $>40 \%$ SerpinA5 expression in serous borderline tumor (a), no expression (b) or focal $(<10 \%)$ (c) SerpinA5 expression in serous carcinoma. This panel also shows SerpinA5 expression in blood vessels, used as internal positive control.

carcinomas $(P=0.038)$. This difference in low-grade and high-grade carcinomas was also detected when the analysis was performed in advanced-stage (FIGO stage III/IV) patients only $(P=0.002)$, whereas SerpinA5 protein expression in early-stage (FIGO stage I/II) patients is similar $(P=0.539)$. In serous borderline tumors, a statistically significant decrease $(P=0.015)$ in SerpinA5 protein expression was observed in advanced versus early stage. In stage I serous borderline tumor patients, 18/22 $(82 \%)$ cases showed $>40 \%$ SerpinA5 expression. Stage III serous borderline tumor patients showed $>40 \%$ SerpinA5 protein expression in $2 / 8(25 \%)$ cases. The reduced SerpinA5 expression level in low-grade serous carcinomas when compared with serous borderline tumors remained statistically different when stratified for early FIGO stage $(P<0.001)$, whereas no difference was detected between advanced-stage serous borderline tumors and advanced-stage low-grade serous carcinomas $(P=0.845)$.

The primary serous carcinoma and matching omental metastasis were available for 88 cases. SerpinA5 staining was successful in $62 / 88$ (71\%) of paired cases. Statistical analysis showed that SerpinA5 protein expression was significantly $(P<0.001)$ higher in primary serous carcinomas when compared with the corresponding metastases (Table 2 and Figures 3a and b). Metastatic tissues showed SerpinA5 protein levels of $<10 \%$ in $45 / 62$ $(73 \%)$, whereas this was $35 / 62(57 \%)$ in primary serous carcinomas. 

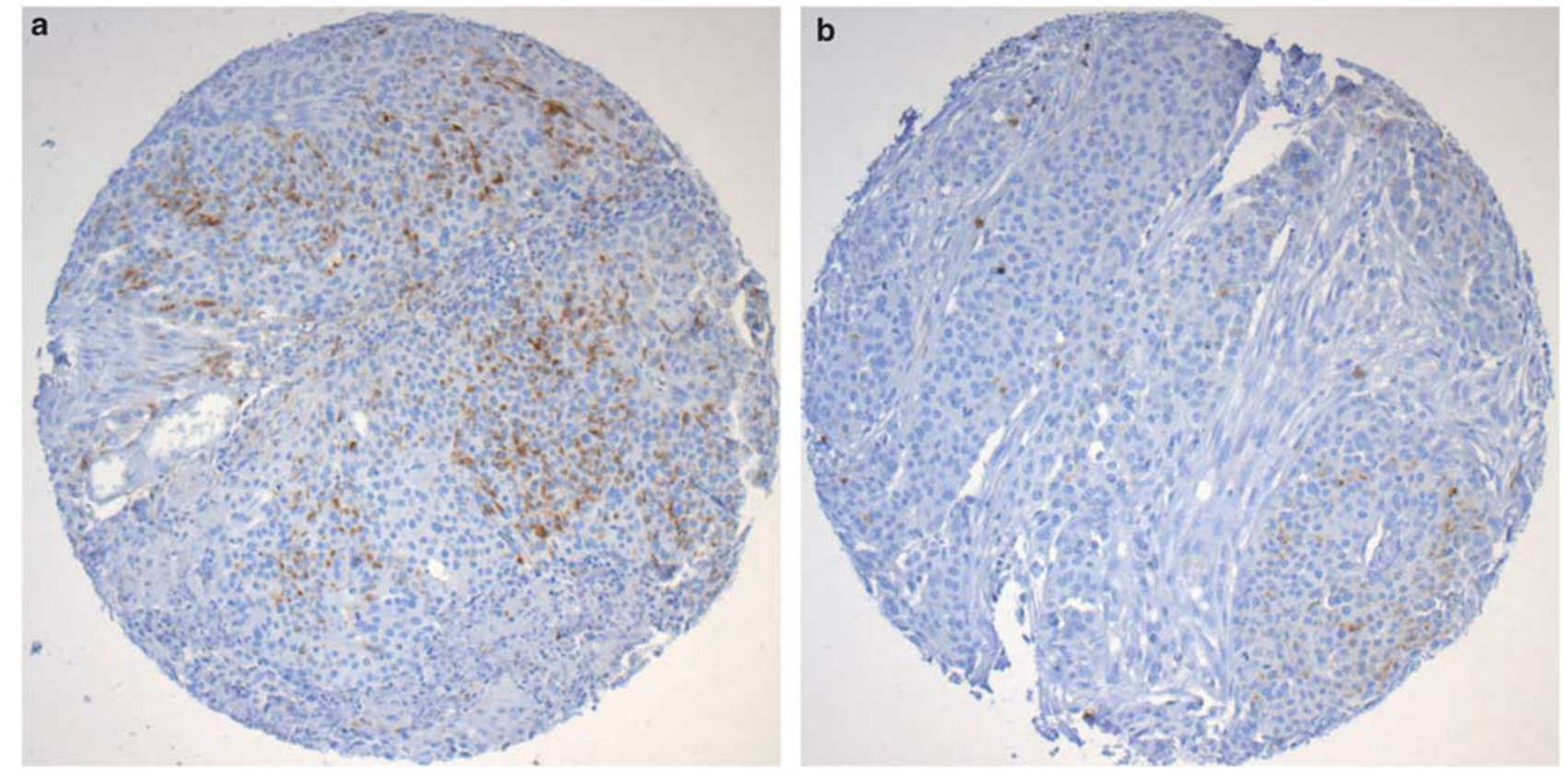

Figure 3 SerpinA5 protein expression is decreased in metastases when compared with primary serous carcinoma. This figure shows SerpinA5 protein expression in a representative primary serous carcinoma (a) and the matching omental metastasis (b).

Kaplan-Meier analysis showed no statistically significant difference in cancer-specific survival in patients with low $(<10 \%)$, moderate $(11-40 \%)$ or high $(>40 \%)$ SerpinA5 protein expression in serous borderline tumors (log-rank; $P=0.649$ ) or carcinomas $(P=0.525$; Maastricht and Groningen series together). Multivariate Cox regression analysis showed that SerpinA5 protein expression was not associated with cause-specific survival, whereas age at diagnosis, FIGO stage and tumor grade were associated with decreased survival.

\section{Discussion}

This study supports other publications ${ }^{19,21}$ that a decrease in SerpinA5 protein expression is associated with invasion. We hypothesized that SerpinA5 protein expression is decreased or lost in serous carcinomas when compared with serous borderline tumors based on mRNA expression results. ${ }^{16}$ In this study we show significantly higher SerpinA5 protein expression in serous borderline tumors when compared with carcinomas $(P<0.001)$. An interesting finding was that SerpinA5 expression was decreased in advanced-stage serous borderline tumors when compared with early-stage borderline tumors. Examination of H\&E slides showed that 6 of the 8 advanced-stage serous borderline tumors demonstrated microinvasion and/or micropapillary architecture, the features of more aggressive borderline tumors. Of these six patients, only one patient had a SerpinA5 protein expression of $>40 \%$. In early-stage serous borderline tumors, microinvasion was not present, and a micropapillary growth pattern was only seen in 4 of 23 cases, however, with SerpinA5 protein expression of $>40 \%$ in all four cases. Decrease or loss of SerpinA5 protein expression might contribute to this microinvasive and/or micropapillary phenotype. Performing a staging procedure in serous borderline tumor cases with lowered SerpinA5 protein expression might thus be recommended.

Comparing serous borderline tumors and lowgrade carcinomas for SerpinA5 protein expression showed a statistically significant reduction in early-stage low-grade carcinomas versus early-stage borderline tumors, whereas advanced-stage serous borderline tumors did not differ from advancedstage low-grade carcinomas. These data indicate that advanced-stage serous borderline tumors are more similar and probably closely related to advancedstage low-grade carcinomas. A drawback of the present study is that the groups are small (8 serous borderline tumors and 18 low-grade carcinomas with advanced-stage disease). Therefore, independent, larger serous borderline tumor and low-grade carcinoma populations are needed to confirm these results. SerpinA5 protein expression differed significantly between low-grade and high-grade serous carcinomas, and although subgroups are small, and data thus have to be interpreted with caution, these data point toward the current literature on low-grade- and high-grade serous carcinomas that low-grade serous carcinomas are more similar to serous borderline tumors than to high-grade serous carcinomas. $^{24}$

Serous carcinomas are defined as invasive based on morphology on H\&E slides, whereas borderline tumors lack destructive stromal invasion. Loss of 
SerpinA5 protein expression might be associated with the invasive properties of serous carcinomas. Extracellular matrix degradation is facilitated by uPA, providing tumor cells to invade surrounding tissue. SerpinA5 is a uPA inhibitor, preventing the conversion of plasminogen to plasmin and subsequent extracellular matrix degradation. ${ }^{25}$ Expression of UPA and downstream activation of MMP9 occur in serous carcinomas that have lost or decreased SerpinA5 protein expression, which is followed by degradation of the extracellular matrix. We and others have shown increased MMP9 levels in serous carcinomas when compared with borderline tumors. ${ }^{16,26}$ Schmalfeldt et $a{ }^{26}$ showed the highest pro-MMP9 levels in metastases. In this study we show that SerpinA5 protein expression was significantly lower in metastatic tissue when compared with the primary tumor. Loss of SerpinA5 expression can contribute to increased MMP activity and subsequent invasive and metastatic potential.

The two main pathways involved in serous ovarian cancer include the RAS signaling pathway and the TP53 pathway. The RAS signaling pathway is constitutively active in $\sim 60 \%$ of serous borderline tumors. ${ }^{9,10}$ We hypothesized that activation of this pathway is associated with SerpinA5 expression to prevent downstream extracellular matrix degradation. ${ }^{16}$ However, BRAF or KRAS mutations were not associated with SerpinA5 protein expression in serous borderline tumors (data not shown). A feedback loop between TP53 and uPA expression has been demonstrated in lung epithelial cells. ${ }^{27,28}$ Reintroduction of TP53 in TP53-deficient lung epithelial cells suppressed uPA expression. PAI-1, a family member of SerpinA5, was shown to be minimally expressed in TP53-deficient lung carcinoma cells. ${ }^{28}$ In serous carcinomas, TP53 mutations occur in up to $80 \%$ of cases. It is reasonable that SerpinA5 is downregulated in serous carcinomas as a result of this mutation. TP53 mutational status was available for serous carcinomas derived from the Maastricht series. Of the 44 serous carcinomas, 24 (59\%) contained a TP53 mutation. A statistically significant difference in SerpinA5 protein expression in relation to TP53 mutation was not found (data not shown).

Although loss of SerpinA5 protein expression seems to be associated with invasiveness, it is not a prognostic factor as survival of serous carcinoma patients was unaffected. These results were also seen for SerpinA1 and TIMP-1 in ovarian cancer. ${ }^{29,30}$ These data indicate that other 'hallmarks' of cancer ${ }^{31}$ in addition to invasion are presumably involved in determining patient outcome.

In conclusion, we showed that SerpinA5 protein expression decreases in advanced stages, invasive serous tumors and the corresponding metastases. Loss of SerpinA5 is associated with micropapillary growth and microinvasion in serous borderline tumors and the invasive phenotype of serous carcinomas.

\section{Acknowledgement}

We thank Thom Boerman from Stichting PAMM, Eindhoven, The Netherlands, for providing material of serous borderline tumors.

\section{Disclosure/conflict of interest}

The authors declare no conflict of interest.

\section{References}

1 Ferlay J, Shin HR, Bray F, et al. Estimates of worldwide burden of cancer in 2008: GLOBOCAN 2008. Int J Cancer 2010;127:2893-2917.

2 Hart WR. Borderline epithelial tumors of the ovary. Mod Pathol 2005;18(Suppl 2):S33-S50.

3 Crum CP, Drapkin R, Miron A, et al. The distal fallopian tube: a new model for pelvic serous carcinogenesis. Curr Opin Obstet Gynecol 2007;19:3-9.

4 Kurman RJ, Shih Ie M. The origin and pathogenesis of epithelial ovarian cancer: a proposed unifying theory. Am J Surg Pathol 2010;34:433-443.

5 Marquez RT, Baggerly KA, Patterson AP, et al. Patterns of gene expression in different histotypes of epithelial ovarian cancer correlate with those in normal fallopian tube, endometrium, and colon. Clin Cancer Res 2005; 11:6116-6126.

6 Prat J. Surface epithelial-stromal tumors: serous tumors. In: Prat J (ed). Pathology of the Ovary. Saunders: Philadelphia, PA, 2004. pp 83-111.

7 Scully RE, Young RH, Clement PB. Surface epithelialstromal tumors and serous tumors In: Rosai J (ed). Tumors of the Ovary, Maldeveloped Gonads, Fallopian Tube, and Broad Ligament. AFIP Atlas of Tumor Pathology, Series III, Fasc.23.\#\#AFIP: Washington DC, 1998;51-80.

8 Cannistra SA. Cancer of the ovary. N Engl J Med 2004; 351:2519-2529.

9 Sieben NL, Macropoulos P, Roemen GM, et al. In ovarian neoplasms, BRAF, but not KRAS, mutations are restricted to low-grade serous tumours. J Pathol 2004;202:336-340.

10 Singer G, Oldt III R, Cohen Y, et al. Mutations in BRAF and KRAS characterize the development of low-grade ovarian serous carcinoma. J Natl Cancer Inst 2003; 95:484-486.

11 Shih Ie M, Kurman RJ. Molecular pathogenesis of ovarian borderline tumors: new insights and old challenges. Clin Cancer Res 2005;11:7273-7279.

12 Bonome T, Lee JY, Park DC, et al. Expression profiling of serous low malignant potential, low-grade, and high-grade tumors of the ovary. Cancer Res 2005; 65:10602-10612.

13 Gilks CB, Vanderhyden BC, Zhu S, et al. Distinction between serous tumors of low malignant potential and serous carcinomas based on global mRNA expression profiling. Gynecol Oncol 2005;96:684-694.

14 Meinhold-Heerlein I, Bauerschlag D, Hilpert F, et al. Molecular and prognostic distinction between serous ovarian carcinomas of varying grade and malignant potential. Oncogene 2005;24:1053-1065.

15 Ouellet V, Provencher DM, Maugard CM, et al. Discrimination between serous low malignant 
potential and invasive epithelial ovarian tumors using molecular profiling. Oncogene 2005;24:4672-4687.

16 Sieben NL, Oosting J, Flanagan AM, et al. Differential gene expression in ovarian tumors reveals Dusp 4 and Serpina 5 as key regulators for benign behavior of serous borderline tumors. J Clin Oncol 2005; 23:7257-7264.

17 Huntington JA, Li W. Structural insights into the multiple functions of protein $\mathrm{C}$ inhibitor. Cell Mol Life Sci 2009;66:113-121.

18 Geiger M. Protein C inhibitor, a serpin with functions in- and outside vascular biology. Thromb Haemost 2007;97:343-347.

19 Wakita T, Hayashi T, Nishioka J, et al. Regulation of carcinoma cell invasion by protein $\mathrm{C}$ inhibitor whose expression is decreased in renal cell carcinoma. Int J Cancer 2004;108:516-523.

20 Cao Y, Becker C, Lundwall A, et al. Expression of protein $\mathrm{C}$ inhibitor (PCI) in benign and malignant prostatic tissues. Prostate 2003;57:196-204.

21 Asanuma K, Yoshikawa T, Hayashi T, et al. Protein C inhibitor inhibits breast cancer cell growth, metastasis and angiogenesis independently of its protease inhibitory activity. Int J Cancer 2007;121:955-965.

22 Castello R, Landete JM, Espana F, et al. Expression of plasminogen activator inhibitors type 1 and type 3 and urokinase plasminogen activator protein and mRNA in breast cancer. Thromb Res 2007;120:753-762.

23 de Graeff P, Hall J, Crijns AP, et al. Factors influencing p53 expression in ovarian cancer as a biomarker of clinical outcome in multicentre studies. Br J Cancer 2006;95:627-633.

24 Kurman RJ, Shih Ie M. Pathogenesis of ovarian cancer: lessons from morphology and molecular biology and their clinical implications. Int J Gynecol Pathol 2008;27:151-160.

25 Smith HW, Marshall CJ. Regulation of cell signalling by uPAR. Nat Rev Mol Cell Biol 2010;11:23-36.

26 Schmalfeldt B, Prechtel D, Harting $\mathrm{K}$, et al. Increased expression of matrix metalloproteinases (MMP)-2, MMP9 , and the urokinase-type plasminogen activator is associated with progression from benign to advanced ovarian cancer. Clin Cancer Res 2001;7:2396-2404.

27 Shetty S, Gyetko MR, Mazar AP. Induction of p53 by urokinase in lung epithelial cells. J Biol Chem 2005; 280:28133-28141.

28 Shetty S, Padijnayayveetil J, Tucker T, et al. The fibrinolytic system and the regulation of lung epithelial cell proteolysis, signaling, and cellular viability. Am J Physiol Lung Cell Mol Physiol 2008;295:L967-L975.

29 Normandin K, Peant B, Le Page C, et al. Protease inhibitor SERPINA1 expression in epithelial ovarian cancer. Clin Exp Metastasis 2010;27:55-69.

30 Steffensen KD, Waldstrom M, Christensen RK, et al. Lack of relationship between TIMP-1 tumour cell immunoreactivity, treatment efficacy and prognosis in patients with advanced epithelial ovarian cancer. BMC Cancer 2010;10:185.

31 Hanahan D, Weinberg RA. The hallmarks of cancer. Cell 2000;100:57-70. 